\title{
Probing photon decay with the Sunyaev-Zel'dovich effect
}

\author{
S. Colafrancesco ${ }^{1,2}$ and P. Marchegiani ${ }^{1,2}$ \\ 1 INAF - Osservatorio Astronomico di Roma via Frascati 33, 00040 Monteporzio, Italy \\ e-mail: sergio.colafrancesco@oa-roma.inaf.it \\ 2 School of Physics, University of the Witwatersrand, Private Bag 3, 2050-Johannesburg, South Africa \\ e-mail: sergio.colafrancesco@wits.ac.za
}

Received 21 November 2013 / Accepted 14 December 2013

\begin{abstract}
Context. The fundamental properties of the photon have a deep impact on the astrophysical processes that involve it, such as the inverse Compton scattering of CMB photon by energetic electrons residing within galaxy cluster atmospheres. This is usually referred to as the Sunyaev-Zel'dovich effect (SZE).

Aims. We calculate the combined constraints on the photon decay time and mass by studying the impact of the modified CMB spectrum on the SZE of galaxy clusters.

Methods. We analyze the modifications of the SZE as produced by photon decay effects. We study the frequency ranges where these modifications are large and where the constraints derived from the SZE are stronger than those already obtained from the CMB spectrum.

Results. We show that the SZE can set limits on the photon decay time and mass, or on $E^{*}=\frac{t_{0}}{\tau_{\gamma}} m_{\gamma} c^{2}$, which are stronger than those obtained from the CMB. The main constraints come from the low-frequency range $v \approx 10-50 \mathrm{GHz}$ where the modified SZE $\Delta I_{\text {mod }}$ is greater than the standard one $\Delta I$, with the difference $\left|\left(\Delta I_{\bmod }-\Delta I\right)\right|$ increasing with the frequency for increasing values of $E^{*}$. Additional constraints can be set in the range $120-180 \mathrm{GHz}$ where there is an increase in the frequency position of the minimum of $\Delta I_{\text {mod }}$ with respect to the standard one with increasing values of $E^{*}$.

Conclusions. We demonstrated that the effect of photon decay can be measured or constrained by the Square Kilometer Array in the optimal range $\approx 10-30 \mathrm{GHz}$ setting limits of $E^{*} \lesssim 1.4 \times 10^{-9} \mathrm{eV}$ and $5 \times 10^{-10} \mathrm{eV}$ for 30- and 260-h integration for A2163, respectively. These limits are tighter than those obtained with the COBE-FIRAS spectral measurements of the CMB.
\end{abstract}

Key words. cosmology: theory - cosmic background radiation - galaxies: clusters: general

\section{Introduction}

Having a non-zero mass for the photon is a valid theoretical possibility described by the Proca (1936) equations that extend Maxwell equations to the case of a massive photon. Even in the context of the quantum theories of electro-magnetic fields, the gauge invariance break caused by a non-zero photon mass can be repaired via the Stückelberg (1957) mechanism.

Experimental limits on the photon mass can be derived from laboratory experiments on the Coulomb law and on magnetic fields (see discussion in Heeck 2013), with more stringent limits that can be derived by using magnetic fields on extra terrestrial scales. In fact, the most stringent limit available today, $m_{\gamma} c^{2}<$ $10^{-18} \mathrm{eV}$, is derived from observations of the magnetic field in the solar wind (Ryutov 2007). Observations of magnetic fields on larger scales can yield even stronger (although still uncertain) limits (see Goldhaber \& Nieto 2010).

More recently Heeck (2013) has also discussed the possibility that a photon might decay in lighter particles (e.g., the lightest neutrino or even particles outside the standard model), showing that - contrary to the limits on the photon mass - there are not yet any stringent experimental limits on the photon decay time. In his work, Heeck (2013) derived a first limit on the photon decay time comparing the distortion on the cosmic microwave background $(\mathrm{CMB})$ spectrum induced by the photon decay with the
COBE-FIRAS data. The constraints derived from the CMB yield fairly weak limits on the photon mass, but very competitive limits on the photon decay time that are stronger than the existing ones:

$\tau_{\gamma}>2 \times 10^{-10}\left(\frac{m_{\gamma} c^{2}}{10^{-18} \mathrm{eV}}\right) t_{0}$

where $t_{0}=13.8 \times 10^{9} \mathrm{yr}$ is the age of the Universe.

In this work we want to explore the possibility of setting even stronger limits on the basic properties of the photon (i.e. its mass and decay time) by using the Sunyaev-Zel'dovich effect (SZE; Sunyaev \& Zel'dovich 1972, see Colafrancesco et al. 2003 for a generalized derivation), which are the spectral distortions produced by the inverse Compton scattering of CMB photons off the high-energy electrons (both thermal and non-thermal) that are present in clusters of galaxies and other cosmic structures (see Birkinshaw 1999; Colafrancesco 2012, for reviews). Even though the SZE in clusters yields a distortion of the CMB spectrum typically of $\sim 10^{-5}$, its ability to derive limits on the photon properties can be favored by the fact that it is a differential measure of spectral CMB distortions; therefore, it can be easier to detect a trace of the photon decay through specific SZE spectral measurements or to set more stringent limits on the photon properties than those obtained from the $\mathrm{CMB}$ spectrum analysis. 


\section{The SZE with decaying CMB photons}

The effect of photon decay on the CMB spectrum can be calculated (see, e.g., Heeck 2013), and the final energy density of the photon population is given by

$$
\begin{aligned}
\rho\left(E, T_{0}\right) \mathrm{d} E \simeq & \frac{8 \pi}{(h c)^{3}} \frac{E^{3} \mathrm{~d} E}{\exp \left(\sqrt{E^{2}-m_{\gamma}^{2} c^{4}} / k_{\mathrm{B}} T_{0}\right)-1} \\
& \times \sqrt{1-\frac{m_{\gamma}^{2} c^{4}}{E^{2}}} \exp \left(-\frac{\Gamma_{0}}{\hbar c} \frac{m_{\gamma} c^{2}}{E} \mathrm{~d}_{L}\right)
\end{aligned}
$$

where $T_{0}$ is the CMB temperature, $\Gamma_{0}=\hbar / \tau_{\gamma}$ is the decay width of the photon, and $d_{L}=47 \times 10^{9}$ ly is the comoving distance of the last scattering surface. In the following we define the quantity

$E^{*} \equiv \frac{\Gamma_{0}}{\hbar} t_{0} m_{\gamma} c^{2}=\frac{t_{0}}{\tau_{\gamma}} m_{\gamma} c^{2}$

that parametrizes the effect of the photon decay. The limits on both the photon mass and its decay time obtained by Heeck (2013), once combined together, provide a limit on the quantity $E^{*}$ of

$E^{*}<5 \times 10^{-9} \mathrm{eV}$.

The spectral distortion of the CMB spectrum due to the SZE is given by

$I(x)=\int_{-\infty}^{+\infty} I_{0}\left(x e^{-s}\right) P(s) \mathrm{d} s$

(Wright 1979; Colafrancesco et al. 2003), where $x=h v /\left(k_{\mathrm{B}} T_{0}\right)$ is the normalized photon frequency, $P(s)$ the photon redistribution function (yielding the probability of a logarithmic shift $s=\ln \left(v^{\prime} / v\right)$ in the photon frequency) that depends on the electron spectrum producing the CMB Comptonization, and $I_{0}(x)$ the specific intensity of the incident $\mathrm{CMB}$ radiation field.

The redistribution function $P(s)$ can be calculated at the desired approximation order in the plasma depth $\tau=\sigma_{T} \int n_{\mathrm{e}} \mathrm{d} \ell$ (where $n_{\mathrm{e}}$ is the plasma electron density) or via a general relativistic method by using Fourier transform properties (see Colafrancesco et al. 2003, for details). Our method contains the relativistic corrections required for high temperature or relativistic electrons (see, e.g., Wright 1979; Rephaeli 1995; Challinor \& Lasenby 1998; Itoh et al. 1998; Sazonov \& Sunyaev 1998), the effect of multiple scattering (see, e.g., Molnar \& Birkinshaw 1999; Dolgov et al. 2001; Itoh et al. 2001), and the treatment of multiple electron population combination.

In the following we calculate the SZE at second order in $\tau$ for the sake of having reasonably short computational time. We have verified, however, that our results do not change appreciably even in the general, exact computation case. We study the case of the SZE produced by a thermal electron population that provides the dominant contribution to the SZE observed in galaxy clusters with respect to the kinematic and non-thermal SZEs (see, e.g., Enßlin \& Kaiser 2000; Colafrancesco et al. 2003). We stress here that our general method also allows considering the additional contributions to the total SZE as produced by the kinematic SZE, and additional non-thermal electron populations, as well as by other contributions to the SZE like lineof-sight variations in electron temperature and density (see, e.g., Colafrancesco \& Marchegiani 2010). We also stress here that Chluba et al. (2014) and Chluba \& Dai (2013) show that the effect of scattering anisotropies of the radiation field needs to be

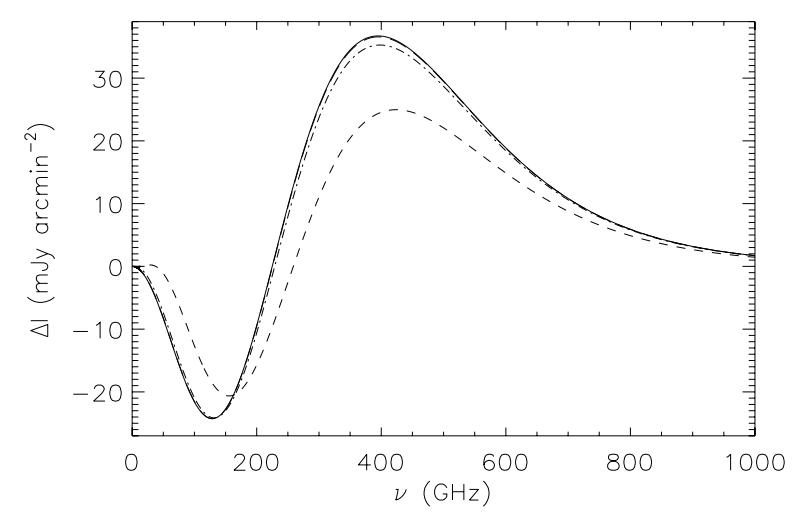

Fig. 1. Thermal SZE (see Eq. (8)) for $k_{\mathrm{B}} T=15 \mathrm{keV}$ and $\tau=1 \times 10^{-2}$ is shown for the case $E^{*}=0$ (solid curve, corresponding to the standard SZE), $10^{-6} \mathrm{eV}$ (long dashed curve), $10^{-5} \mathrm{eV}$ (dot-dashed curve), and $10^{-4} \mathrm{eV}$ (dashed curve).

included to obtain correct results already at second order in $\tau$. We discuss in Sect. 3 the impact of the scattering anisotropies of the radiation field on our results.

The spectrum of the incident CMB radiation field $I_{0}(x)$ is derived from Eq. (2) which describes the CMB energy density modified by the photon decay process

$$
\begin{aligned}
I_{0, \text { mod }}(x)= & 2 \frac{\left(k_{\mathrm{B}} T_{0}\right)^{3}}{(h c)^{2}} \frac{x^{3}}{\exp \left[\sqrt{E^{2}-m_{\gamma}^{2} c^{4}} / E_{0}\right]-1} \\
& \times \sqrt{1-\frac{m_{\gamma}^{2} c^{4}}{E^{2}}} \exp \left[-\frac{E^{*}}{E} \frac{d_{L}}{c t_{0}}\right]
\end{aligned}
$$

where $E_{0}=k_{\mathrm{B}} T_{0}$ and $E=h v=x E_{0}$. We note that for $m_{\gamma}=0$, which yields the value $E^{*}=0$, one obtains the original CMB spectrum without the photon decay effect:

$I_{0}(x)=2 \frac{\left(k_{\mathrm{B}} T_{0}\right)^{3}}{(h c)^{2}} \frac{x^{3}}{\mathrm{e}^{x}-1}$.

Inserting the incoming modified CMB spectrum of Eq. (6) in (5), we obtain the SZE spectrum modified by the photon decay process as

$\Delta I_{\text {mod }}(x)=I_{\text {mod }}(x)-I_{0, \bmod }(x)$

which can be calculated as a function of the relevant parameters $m_{\gamma}$ and $\tau_{\gamma}$. In the following we use, for simplicity, the single parameter $E^{*}$ to quantify the changes in the SZE and in the CMB spectrum due to the photon decay. We note that in the non-relativistic limit and for low values of the electron temperature $T$ and optical depth $\tau$, the SZE spectrum in Eq. (8) can be expressed in the form $\Delta I_{\text {mod }}(x)=2\left[\left(k_{\mathrm{B}} T_{0}\right)^{3} /(h c)^{2}\right] y_{0} g_{\text {mod }}(x)$, where $y_{0}=\sigma_{T} \int\left(k_{\mathrm{B}} T / m_{\mathrm{e}} c^{2}\right) n_{\mathrm{e}} \mathrm{d} \ell$, and the function $g_{\text {mod }}(x)$ is given at the first order approximation in the parameter $\epsilon \equiv$ $m_{\gamma} c^{2} / E_{0} \ll 1$ as

$$
\begin{aligned}
g_{\text {mod }}(x)= & g(x)+\epsilon \frac{d_{L}}{c \tau_{\gamma}}\left[\frac{x^{3}}{e^{x}-1}\right]\left[\frac{e^{x}(2+x)}{e^{x}-1}\right. \\
& \left.+-\frac{2 x e^{2 x}}{\left(e^{x}-1\right)^{2}}+\frac{2}{x}\right]
\end{aligned}
$$

where $g(x)$ is the non-relativistic SZE spectral function (see Zel'dovich \& Sunyaev 1969).

Figure 1 shows the spectrum of the thermal SZE for an electron population in a cluster with a temperature $k_{\mathrm{B}} T=15 \mathrm{keV}$ 

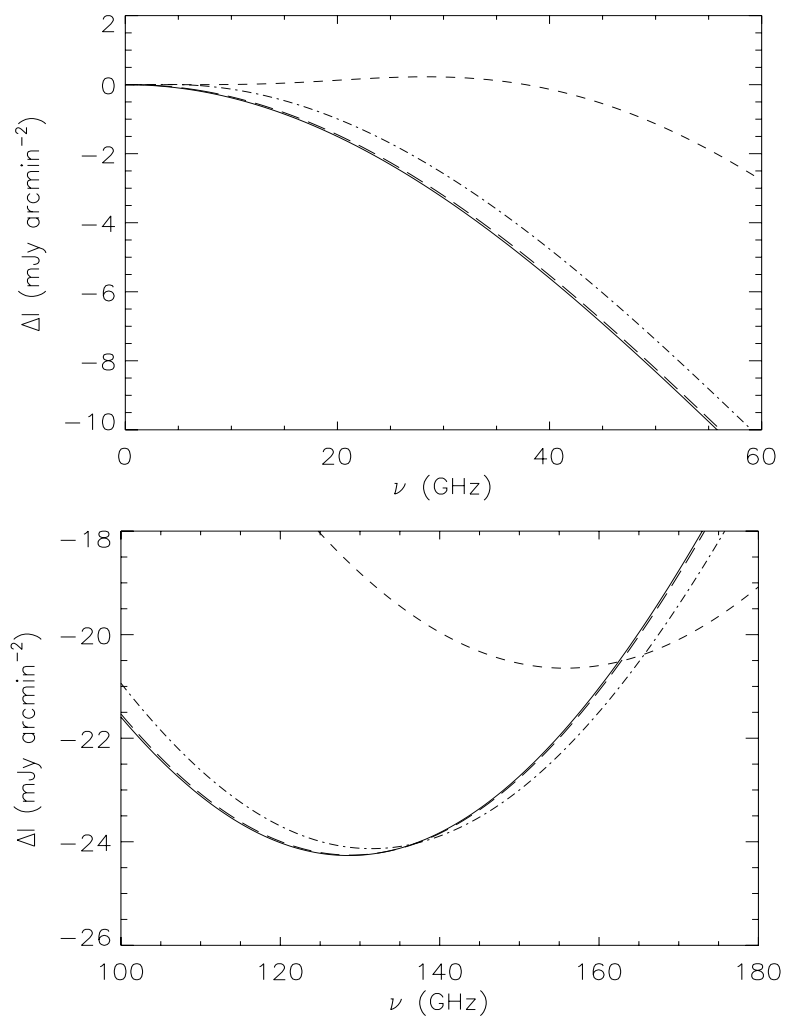

Fig. 2. Zoom of the plot in Fig. 1 for the two most interesting spectral regions (see text for details).

and depth $\tau=1 \times 10^{-2}$ for various values of $E^{*}$. Figure 2 shows a zoom of the curves in Fig. 1 in some interesting spectral regions: the low $-v$ region $(0-60 \mathrm{GHz})$ and the frequency region around the minimum of the SZE in the range 100-180 GHz. These spectral regions are interesting because there is a significant difference between the standard SZE and the one modified by the photon decay that becomes more evident with increasing values of $E^{*}$. It is clear, however, that the limit set by Eq. (4) indicates that the SZE distortion must be smaller than the standard amplitude of the SZE, so it also indicates that we need a more detailed theoretical analysis and a wider exploration of experimental techniques to determine the effect of photon decay on the SZE. In the next section we more specifically discuss the possibilities of detecting the variations in the thermal SZE due to the photon decay for various values of the parameter $E^{*}$.

\section{Discussion}

Figures 1 and 2 show that the distortion of the thermal SZE induced by the photon decay has a peculiar spectral characteristic. At high frequencies $(v \gtrsim 220 \mathrm{GHz})$ this distortion is similar to that of a non-thermal SZE (i.e., a shift in the zero and the maximum of the SZE towards high frequencies, so it is difficult to disentangle; see, e.g., Enßlin \& Kaiser (2000), Colafrancesco et al. (2003, 2009), Colafrancesco \& Marchegiani (2010), Colafrancesco et al. (2011). At low frequencies it shows a quite unique spectral behavior, i.e., a shift of the minimum of the SZE towards higher frequencies and a very unique spectral shape at very low frequencies where the modified SZE can be positive in sign. (We remind the reader that the standard SZE is always negative at all frequencies below the zero, i.e. at $v \lesssim 220 \mathrm{GHz}$.) Therefore, observations of the SZE at very low frequencies (i.e., $v \lesssim 50 \mathrm{GHz}$ ) and/or around the minimum (i.e.,
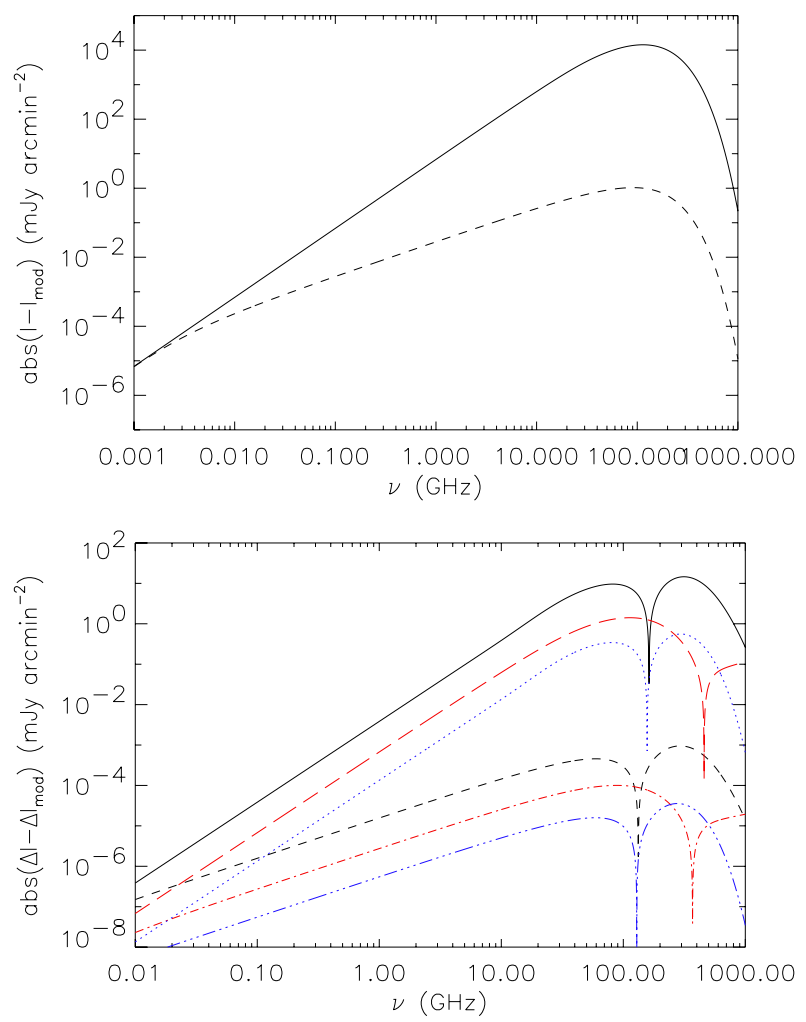

Fig. 3. Upper panel: absolute difference between the standard CMB spectrum and the ones modified for $E^{*}=10^{-4} \mathrm{eV}$ (solid curve) and for $E^{*}=5 \times 10^{-9} \mathrm{eV}$ (dashed curve). Lower panel: absolute difference between the standard thermal SZE for $k T=15 \mathrm{keV}$ and $\tau=1 \times 10^{-2}$ and the ones modified with $E^{*}=10^{-4} \mathrm{eV}$ (solid curve) and for $E^{*}=5 \times 10^{-9} \mathrm{eV}$ (dashed curve), between the standard non-thermal SZE (Colafrancesco et al. 2003) for $p_{1}=10, s=2.7$, and $\tau=1 \times 10^{-4}$ and the ones modified with $E^{*}=10^{-4} \mathrm{eV}$ (red, long-dashed curve) and for $E^{*}=5 \times 10^{-9} \mathrm{eV}$ (red, dot-dashed curve), and between the standard non-relativistic SZE for $y_{0}=1 \times 10^{-5}$ and the ones modified with $E^{*}=10^{-4} \mathrm{eV}$ (blue, dotted curve) and for $E^{*}=5 \times 10^{-9} \mathrm{eV}$ (blue, three-dot-dashed curve).

in the range $100-150 \mathrm{GHz}$ ) can provide crucial information on a possible photon decay effect.

Figure 3 shows a comparison between the difference of the standard and modified CMB spectrum (for two different values of $E^{*}$ ) and the difference between the relative SZE spectra in a thermal and a non-thermal case, as well as in the nonrelativistic case. For the CMB spectrum, the maximum difference is found around $100 \mathrm{GHz}\left(114\right.$ and $90.5 \mathrm{GHz}$ for $E^{*}=10^{-4}$ and $5 \times 10^{-9} \mathrm{eV}$, where the difference between the CMB spectra is $1.43 \times 10^{4}$ and $1.04 \mathrm{mJy} \operatorname{arcmin}^{-2}$, respectively). For the thermal SZE, the maximum difference is found in two distinct spectral regions: 82.0 and $314 \mathrm{GHz}$ for $E^{*}=10^{-4} \mathrm{eV}$, and 59.5 and $292 \mathrm{GHz}$ for $E^{*}=5 \times 10^{-9} \mathrm{eV}$, where the differences between the SZE spectra are 9.60 and $14.6 \mathrm{mJy} \operatorname{arcmin}^{-2}$ for $E^{*}=$ $10^{-4} \mathrm{eV}$, and $4.56 \times 10^{-4}$ and $9.50 \times 10^{-4} \mathrm{mJy} \mathrm{arcmin}^{-2}$ for $E^{*}=$ $5 \times 10^{-9} \mathrm{eV}$. For the non-thermal SZE the frequencies at which the maximum difference is found are 113 and $2.86 \times 10^{4} \mathrm{GHz}$ for $E^{*}=10^{-4} \mathrm{eV}$ and 88.4 and $1.96 \times 10^{4} \mathrm{GHz}$ for $E^{*}=5 \times 10^{-9} \mathrm{eV}$, where the differences are 1.41 and $0.675 \mathrm{mJy} \mathrm{arcmin}^{-2}$, and $1.00 \times 10^{-4}$ and $5.79 \times 10^{-5} \mathrm{mJy} \operatorname{arcmin}^{-2}$, respectively. To set more stringent constraints than the available ones on the photon decay, it is necessary to have an instrumental sensitivity below these values in the two interesting frequency windows. 

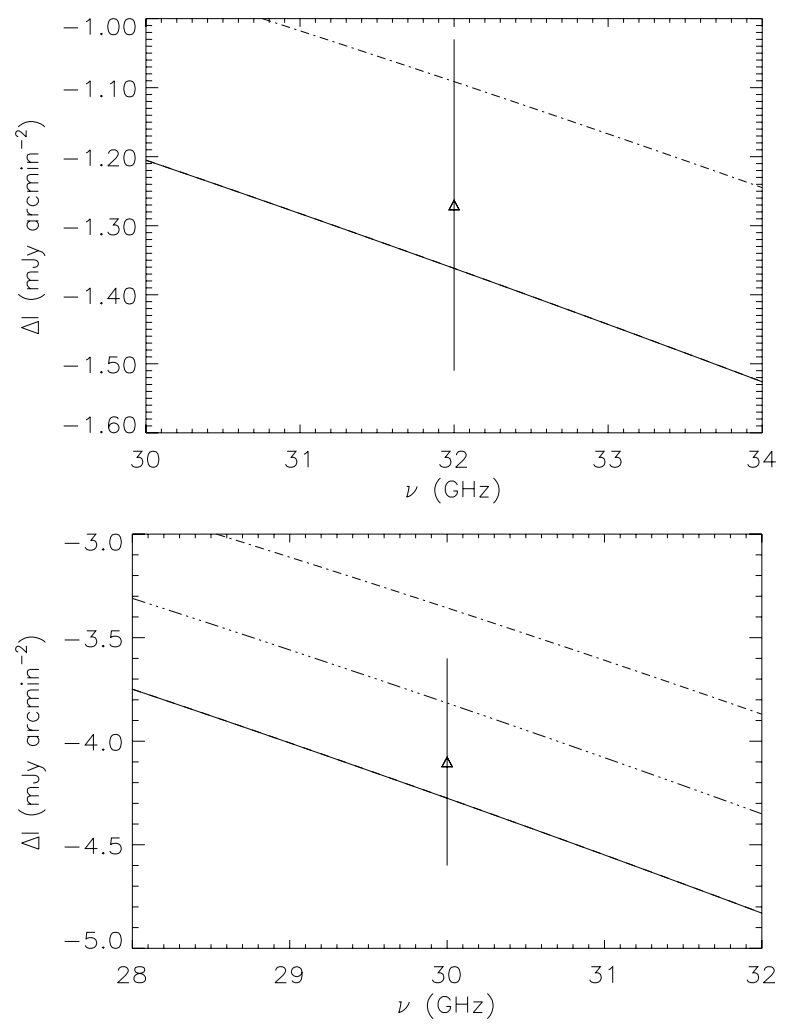

Fig. 4. Upper panel: SZE calculated for Coma (with $k T=8.2 \mathrm{keV}$ and $\tau=6.55 \times 10^{-3}$ ) for $E^{*}=0$ (solid), $5 \times 10^{-9}$ (dashed), and $1 \times$ $10^{-5}$ (dot-dashed) eV. Lower panel: SZE calculated for A2163 (with $k T=12.4 \mathrm{keV}$ and $\tau=1.56 \times 10^{-2}$ ) for $E^{*}=0$ (solid), $5 \times 10^{-9}$ (dashed), $5 \times 10^{-6}$ (3 dot-dashed), and $1 \times 10^{-5}$ (dot-dashed) $\mathrm{eV}$. The Coma parameters are taken from Hughes et al. (1989) and Briel et al. (1992), and from Elbaz et al. (1995) and Markevitch et al. (1996) for A2163.

The CMB spectral distortion induced by the photon decay leads to a shift of the SZE minimum towards high frequencies. This effect is a unique signature of the photon decay because the position of the SZE minimum depends very weakly on any other additional astrophysical effects, such as the nonthermal SZE (see, e.g., Enßlin \& Kaiser 2000; Colafrancesco et al. 2003; Colafrancesco \& Marchegiani 2010). We studied the frequency shift of the SZE minimum $v_{\text {min }}$ from its standard value of $\approx 128 \mathrm{GHz}$ with increasing $E^{*}$. We found that this effect becomes evident only for $E^{*}>10^{-5} \mathrm{eV}$, which is much higher than the limit $E^{*}<5 \times 10^{-9} \mathrm{eV}$ derived from CMB spectral measurements (Heeck 2013): the minimum frequency is 129, 131, 143 , and $156 \mathrm{GHz}$ for $E^{*}=10^{-6}, 10^{-5}, 5 \times 10^{-5}$, and $10^{-4} \mathrm{eV}$, respectively.

Figure 4 shows the comparison between the thermal SZE in the Coma and A2163 clusters calculated for various values of $E^{*}$ and the available SZE data from OVRO (for Coma; Herbig et al. 1995) and OVRO/Bima (for A2163 LaRoque et al. 2002). The OVRO data allows setting limits $E^{*} \lesssim 7 \times 10^{-6} \mathrm{eV}$ for A2163 and $E^{*} \lesssim 1.2 \times 10^{-5} \mathrm{eV}$ for Coma. We conclude that the uncertainties of the OVRO data are much larger than the precision needed to set constraints on $E^{*}$ stronger or comparable to those of COBE-FIRAS on the CMB spectrum. Figure 5 shows the difference between the standard thermal SZE predicted for A2163 and the one modified by photon decay compared with the expected sensitivity of the Square Kilometer Array (SKA) for different values of $E^{*}$. We found that in $30 \mathrm{~h}$ the SKA is able to measure the SZE difference at the 3.68, 2.21, 1.47, and $0.74 \sigma$

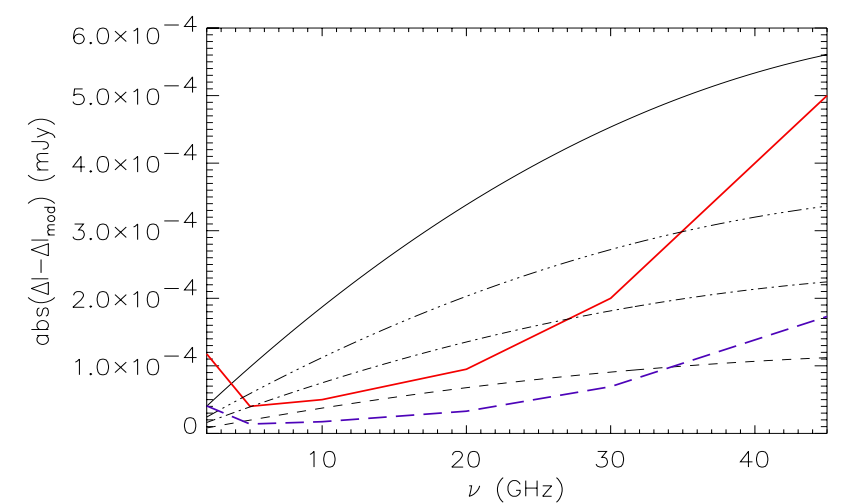

Fig. 5. Difference between the standard thermal SZE calculated for A2 163 in $2-45 \mathrm{GHz}$ range and integrated in a $1 \operatorname{arcmin}^{2}$ area and the one modified for values $E^{*}=5 \times 10^{-9} \mathrm{eV}$ (solid line), $3 \times 10^{-9} \mathrm{eV}$ (dash -3 dots), $2 \times 10^{-9} \mathrm{eV}$ (dot-dashed), and $1 \times 10^{-9} \mathrm{eV}$ (dashed). The sensitivity achievable with SKA in $\sim 30 \mathrm{~h}$ (red thick solid line) and in $\sim 260 \mathrm{~h}$ (blue thick long-dashed line) are shown (see Carilli 2005).

confidence level, and in $260 \mathrm{~h}$ it can measure a difference at the $10.6,6.39,4.26$, and $2.13 \sigma$ level confidence level for $E^{*}=5,3$, 2 , and $1 \times 10^{-9} \mathrm{eV}$, respectively, or to put much more stringent upper limits on the value of $E^{*} \lesssim 1.4 \times 10^{-9} \mathrm{eV}$ and $5 \times 10^{-10} \mathrm{eV}$ for 30 and $260 \mathrm{~h}$, respectively, and consequently set much more stringent constraints on the mass and decay time of the photon. The most effective spectral window for performing such measurements with the SKA is the one between 10 and $30 \mathrm{GHz}$. This frequency band is also advantageous because at these frequencies the synchrotron emission from radio halo diffuse emission in clusters (that has a quite steep spectrum, e.g. $\alpha_{r} \sim 1.2$ for A2163) is particularly low and hence does not contaminate the SZE measurement significantly. The precise knowledge of the radio synchrotron spectrum at low and mid frequencies obtainable with the SKA will also allow its contribution at higher frequencies to be estimated where the modified SZE is more evident. This contribution is at least a conservative upper limit estimate because there is evidence that the radio halo spectrum steep ends at high frequencies (see, e.g., Thierbach et al. 2003, for the case of Coma). The subtraction of this synchrotron emission thus provides a conservative estimate (lower limit) of the distortion of the thermal SZE produced by the photon decay.

Our analysis has focused on the effects of photon decay on the thermal SZE as computed in our general approach (Colafrancesco et al. 2003), leaving the discussion of the cluster peculiar motion effects or effects induced by line-of-sight variation in electrons properties to a later work. We note here that the corrections to the second-order thermal SZE produced by the scattering induced anisotropies (see Chluba et al. 2014) can be at most about three or four times larger than the correction calculated in our approach. Because the second-order correction to the SZE is of order $\tau^{2}$, even for a fairly high value of $\tau \sim 10^{-2}$, the relative change in the total SZE spectrum is at most $\sim 4 \times 10^{-4}$. In addition, we note that this correction becomes smaller for high electron temperatures (Chluba \& Dai 2013) and that the difference with our second-order correction calculation is maximum around the minimum and the maximum regions of the SZE (Chluba et al. 2014). Since we found that high-temperature clusters, like A2163, and the low-frequency band $v \sim 10-50 \mathrm{GHz}$ provide the best cases for probing the photon decay effect via the SZE, we can estimate that these last corrections do not affect our results appreciably. Finally, we stress that our main conclusions are obtained from the study of the difference between the original and the modified SZE, and the difference plotted in Fig. 5 is 
practically unaffected because the scattering-induced anisotropy corrections reflect on both the original and the modified spectra.

\section{Conclusions}

We have demonstrated in this paper that photon decay effects can be effectively studied by using the spectral distortions of the SZE in galaxy clusters observable with the coming highsensitivity radio telescopes like SKA. We showed that measurements of the SZE in the range $\approx 10-50 \mathrm{GHz}$ are more competitive than $\mathrm{CMB}$ spectral measurements for setting stringent constraints on the photon decay time, provided that we reach instrumental sensitivities on the order of $\lesssim 0.1 \mu \mathrm{Jy}$. This frequency band is also the one less affected by other sources of astrophysical contamination, and it will be best explored with the advent of the high-sensitivity SKA telescope. At higher frequencies (i.e., $\sim 120-180$ and $\sim 200-300 \mathrm{GHz}$ ), there are other spectral windows where the SZE method is again competitive if not advantageous compared to the CMB studies. The necessary sensitivity in the high-frequency range can be achieved with the next coming Millimetron space mission (see, e.g., Colafrancesco 2012) even though other astrophysical sources of contamination (e.g., sources of non-thermal SZE and/or special distortions of the SZE due to multiple-temperature regions) could contaminate these measurements (see, e.g., Colafrancesco et al. 2011; and Prokhorov et al. 2012, for the case of the Bullet Cluster). The complementarity between SKA and Millimetron SZE measurements will provide further and more stringent multifrequency constraints on the effect of photon decay in the universe. The approved construction of both SKA and Millimetron will make these measurements realistic in the next few years.

Acknowledgements. S.C. acknowledges support by the South African Research Chairs Initiative of the Department of Science and Technology and National
Research Foundation and by the Square Kilometre Array (SKA). P.M. acknowledges support from the SKA post-graduate bursary initiative.

\section{References}

Birkinshaw, M. 1999, Phys. Rep., 310, 97

Briel, U. G., Henry, J. P., \& Boehringer, H. 1992, A\&A, 259, L31

Carilli, C. 2005, SKA Memo 70,

http: //www . skatelescope.org/publications/

Challinor, A., \& Lasenby, A. 1998, ApJ, 499, 1

Chluba, J., \& Dai, L. 2013, MNRAS, in press [arXiv: 1309. 3274]

Chluba, J., Dai, L., \& Kamionkowski, M. 2014, MNRAS, 437, 67

Colafrancesco, S. 2012, in Frontier Objects in Astrophysics and Particle Physics, Mem. SAIt, in press

Colafrancesco, S., \& Marchegiani, P. 2010, A\&A, 520, A31

Colafrancesco, S., Marchegiani, P., \& Palladino, E. 2003, A\&A, 397, 27

Colafrancesco, S., Prokhorov, D. A., \& Dogiel, V. 2009, A\&A, 494, 1

Colafrancesco, S., Marchegiani, P., \& Buonanno, R. 2011, A\&A, 527, L1

Dolgov, A. D., Hansen, S. H., Pastor, S., \& Semikoz, D. V. 2001, ApJ, 554, 74

Elbaz, D., Arnaud, M., \& Bohringer, H. 1995, A\&A, 293, 337

Enßlin, T. A., \& Kaiser, C. R. 2000, A\&A, 360, 417

Goldhaber, A. S., \& Nieto, M. M. 2010, Rev. Mod. Phys., 82, 939

Heeck, J. 2013, Phys. Rev. Lett., 111, 021801

Herbig, T., Lawrence, C. R., \& Readhead, A. C. S. 1995, ApJ, 449, L5

Hughes, J. P. 1989, ApJ, 337, 21

Itoh, N., Kohyama, Y., \& Nozawa, S. 1998, ApJ, 502, 7

Itoh, N., Kawana, Y., Nozawa, S., \& Kohyama, Y. 2001, MNRAS, 327, 567

LaRoque, S. J., Carlstrom, J. E., Reese, E. D., et al. 2002

[arXiv: astro-ph/0204134]

Markevitch, M., Mushotzky, R., Inoue, H., et al. 1996, ApJ, 456, 437

Molnar, S. M., \& Birkinshaw, M. 1999, ApJ, 523, 78

Proca, A. 1936, J. Phys. Radium, 7, 347

Prokhorov, D. A., Million, E. T., Akahori, T., et al. 2012, MNRAS, 426, 2291

Rephaeli, Y. 1995, ApJ, 445, 33

Ryutov, D. D. 2007, Plasma Phys. Control. Fusion, 49, B429

Sazonov, S. Y., \& Sunyaev, R. A. 1998, ApJ, 508, 1

Stückelberg, E. C. G. 1957, Helv. Phys. Acta, 30, 209

Sunyaev, R. A., \& Zel'dovich, Y. B. 1972, A\&A, 20, 189

Thierbach, M., Klein, U., \& Wielebinski, R. 2003, A\&A, 397, 53

Wright, E. L. 1979, ApJ, 232, 348

Zel'dovich, Y. B., \& Sunyaev, R. A. 1969, Ap\&SS, 4, 301 